\title{
Soft theorems in superstring theory
}

\section{Ashoke Sen}

Harish-Chandra Research Institute, ${ }^{1}$

Chhatnag Road, Jhusi, Allahabad 211019, India

E-mail: sen@mri.ernet.in

ABSTRACT: We use insights from superstring field theory to prove the subleading soft graviton theorem for tree amplitudes of (compactified) heterotic and type II string theories for arbitrary number of finite energy NS (NSNS) sector external states but only one soft graviton. We also prove the leading soft graviton theorem in these theories for arbitrary number of external soft gravitons. In our analysis there is no restriction on the mass and spin of the finite energy external states. This method can also be used to give a proof of the subleading soft graviton theorem for tree amplitudes in quantum field theories coupled to gravity with generic interactions. We discuss the technical issue involved in extending this analysis to loop amplitudes of superstring theory including Ramond sector external states, and its possible resolution.

KEYwORDs: String Field Theory, Superstrings and Heterotic Strings

ArXiv EPRINT: 1702.03934

\footnotetext{
${ }^{1}$ Harish-Chandra Research Institute is a Constituent Unit of the Homi Bhabha National Institute, Training School Complex, Anushakti Nagar, Mumbai 400085, India.
} 


\section{Contents}

1 Introduction 1

2 Warm up with scalar field coupled to gravity 3

3 One soft graviton theorem in tree level superstring field theory 6

4 Multiple soft gravitons $\quad 10$

5 Generalizations $\quad 12$

\section{Introduction}

Study of soft graviton theorem has a long history, both in quantum field theories [1-31] and in (super)string theories [32-44]. The recent interest in soft graviton theorem has its origin in the connection between soft theorems and BMS symmetry [45-53].

Our goal in this paper will be to prove certain soft graviton theorems for the tree amplitudes in heterotic and type II string theories - collectively called superstring theory - possibly compactified on certain manifolds. We shall use the language of superstring field theory [54] in which the amplitudes of superstring theory are given as sum of Feynman diagrams just as in ordinary quantum field theories. However we shall not use many details of the theory, and for this reason our analysis will apply also to quantum field theories.

The general strategy for computing an amplitude with soft insertions will be as follows. First we need to identify the Feynman diagrams that give the desired contribution. Then in order to evaluate these Feynman diagrams we have to find the interaction vertices that couple a soft graviton to the rest of the fields. Once the interaction vertices are found we then use them to evaluate the relevant diagrams. The main simplification in our analysis will be in the second step. We follow the following procedure for determining the coupling of a soft graviton to the rest of the fields.

1. Let $\mu, \nu$ denote the coordinate index along the flat non-compact directions. We take the metric $g_{\mu \nu}$ to be sum of three parts: the background $\eta_{\mu \nu}$, the finite energy part $2 h_{\mu \nu}$ and the soft part $2 S_{\mu \nu}$.

2. In our analysis we treat the finite energy and the soft parts of the metric differently. This is certainly possible for tree amplitudes since soft gravitons appear only as external particles and in any Feynman diagram there is a clear distinction between which lines are soft and which lines carry finite energy. ${ }^{1}$

\footnotetext{
${ }^{1}$ For loop amplitudes we can first compute the gauge invariant one particle irreducible (1PI) effective action without this decomposition into soft and finite energy parts and then, while computing the full
} 
3. We first set $S_{\mu \nu}$ to zero and expand the action in powers of $h_{\mu \nu}$ and other fields around the vacuum solution. The resulting action has manifest Lorentz invariance but not manifest general coordinate invariance.

4. We gauge fix this action by using a Lorentz covariant gauge fixing condition.

5. In the resulting action we now replace $\eta_{\mu \nu}$ by $\eta_{\mu \nu}+2 S_{\mu \nu}$, and all derivatives by covariant derivatives computed using the Christoffel connection of the metric $\eta_{\mu \nu}+$ $2 S_{\mu \nu}$. By expanding the resulting action to first order in $S_{\mu \nu}$ we determine the coupling of the soft graviton to the rest of the fields. ${ }^{2}$

6. This generates the coupling of the soft graviton to the rest of the fields, including finite energy components of the metric, up to first order in the derivatives but misses the terms involving two or more derivatives of $S_{\mu \nu}$ from possible couplings via the Riemann tensor computed from $\eta_{\mu \nu}+2 S_{\mu \nu}$. Therefore our results are valid to first subleading order in the momentum of the soft particle.

7. This action is invariant under general coordinate transformation of $S_{\mu \nu}$. But since $S_{\mu \nu}$ will only appear as external line, we do not need to fix any gauge for $S_{\mu \nu}$. Indeed we only make use of part of this action containing terms linear in $S_{\mu \nu}$ and not the full action.

8. We can now replace $S_{\mu \nu}$ by $\varepsilon_{\mu \nu} e^{i k . x}$ to determine the coupling of a soft graviton of momentum $k$ and polarization $\varepsilon$. Since the coupling is determined by replacing $\eta_{\mu \nu}$ by $\eta_{\mu \nu}+2 S_{\mu \nu}$, it makes computing the effect of soft graviton coupling simple. For example if we consider part of an amplitude that has a finite limit when the momentum of the soft graviton goes to zero, and if we want to compute just the leading term of this component in the power series expansion in $k$, we simply have to study the effect of replacing $\eta_{\mu \nu}$ by $\eta_{\mu \nu}+2 \varepsilon_{\mu \nu}$. Instead of studying its effect on each vertex and internal propagator, we can determine the result by making this replacement in the final expression for the original amplitude without the soft graviton.

Using this method, we prove that at tree level the subleading soft graviton theorem given in $[6,45,46]$ holds for one soft graviton and arbitrary number of finite energy external states coming from the NS sector in the heterotic string theory and the NSNS sector in the type II string theory. There is no restriction on the mass and spin of the finite energy external states in either analysis. We also generalize the leading order result to the case where there are multiple soft gravitons. We believe that it should be possible to use the method suggested here to prove the subleading soft graviton theorem for string loop amplitudes and Ramond sector external states as well. We discuss in section 5 the

Green's functions by summing over tree amplitudes using the 1PI effective action, use this decomposition. As long as the number of non-compact dimensions is sufficiently high so that infrared and collinear divergences are absent, this is a well defined procedure. However there are other difficulties with loop amplitudes as discussed in section 5, and for this reason we postpone discussion of loop amplitudes to a future publication.

${ }^{2}$ Since we shall apply this procedure on the gauge fixed action, this makes the gauge fixing terms for the finite energy fields covariantized with respect to the soft graviton. 
main technical difficulty in proving this general result. However, since this method misses terms involving Riemann tensor of the soft graviton, which contains two powers of the soft momentum, it cannot be used to express the subsubleading soft graviton amplitude just in terms of the amplitude without the soft graviton. We must separately take into account the effect of the extra terms proportional to the Riemann tensor of the soft graviton, and such terms vary from one theory to another. This is consistent with the fact that subsubleading soft graviton amplitudes are known to be non-universal [43] due to non-minimal coupling of the metric via the Riemann tensor.

Our method can also be generalized to derive the soft photon theorem [55] using the same principle: the coupling of a soft photon to the rest of the fields is determined by making all derivatives into covariant derivatives using the soft photon field. In this case only the leading soft photon theorem is universal since subleading coupling of soft photons can be modified via non-minimal coupling involving field strength.

The rest of the paper is organized as follows. In section 2 we consider a scalar field theory coupled to gravity in arbitrary dimensions with arbitrary interactions, and show how in this theory we can derive the subleading soft graviton theorem for tree level amplitudes with one external soft graviton and arbitrary number of finite energy external scalars. In section 3 we generalize this method to prove subleading soft graviton theorem for tree amplitudes in superstring theory with one external soft graviton and arbitrary number of finite energy external states from the NS sector. In section 4 we extend the result of section 3 to the case where there are multiple soft gravitons, but work only to the leading order in the soft momentum. In section 5 we extend the results to leading soft photon theorem, and also discuss the possible ways of extending the results to loop amplitudes in superstring theory.

\section{Warm up with scalar field coupled to gravity}

In this section we shall consider a theory of a scalar field $\phi$ coupled to gravity in $D$ spacetime dimensions, providing a proof of soft graviton theorem that is slightly different from the one given e.g. in $[13,14]$. The scalar field can be massive or massless. Furthermore, we shall not put any restriction on the interactions, except the requirement of invariance under general coordinate transformation. We shall use the convention that an external or internal massless particle in a Feynman diagram will be called soft if all the components of its momentum are small in the center of momentum frame. On the other hand if an internal particle carries a momentum $p$ that has one or more components large but $p^{2}+M^{2}$, where $M$ denotes the mass of the particle, is small then it will be called a nearly on-shell particle. Finally if $p^{2}+M^{2}$ is of order unity or larger, then it will be called a hard particle.

Let us now consider a tree amplitude where one external soft graviton carrying momentum $k$ is attached to a graph with $N$ external on-shell scalar particles carrying finite momenta $p_{1}, \ldots, p_{N}$. All external momenta will be taken to be ingoing in the Feynman diagram, i.e. outgoing particles will carry negative $p^{0}$. Our goal will be to compute the $k$ dependence of the amplitude to order unity. The leading contribution to this amplitude, of order $k^{-1}$, comes from the diagram shown in figure 1 . In this figure the thick lines 


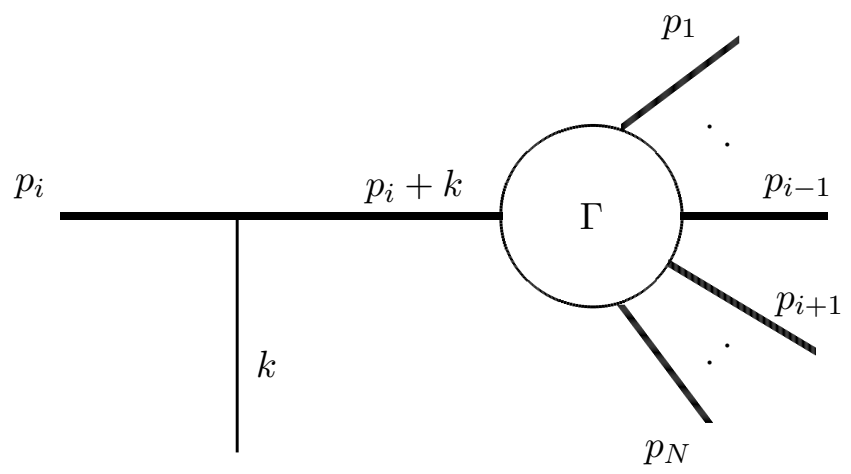

Figure 1. Source of the leading contribution to the amplitude with one external soft graviton.

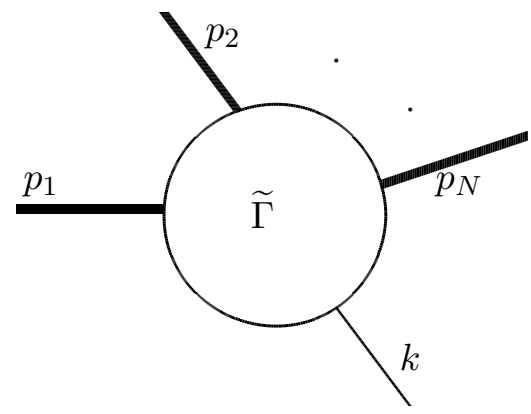

Figure 2. Source of the subleading contribution to the amplitude with one external soft graviton. Here $\widetilde{\Gamma}$ denotes tree amplitudes with external propagators removed, and also diagrams of the type given in figure 1, where the soft graviton attaches to one of the external finite energy lines, removed.

denote either external on-shell finite energy particles or internal nearly on-shell particles, whereas the thin line denotes the external soft particle. $\Gamma$ denotes amputated tree level Green's function - Green's functions from which the external propagators are removed. If $M$ denotes the mass of the scalar field, then for small $k$ the internal propagator carrying momentum $p_{i}+k$ can generate a large factor proportional to

$$
\left\{\left(p_{i}+k\right)^{2}+M^{2}\right\}^{-1}=\left(2 p_{i} \cdot k\right)^{-1} .
$$

It is easy to see that the diagrams given in figure 1 are the only ones that can produce a soft factor in the denominator. At the first subleading order, i.e. at order unity, the contribution to the amplitude comes from the subleading contributions from figure 1 , as well as the leading contribution from the diagram shown in figure 2 .

In order to compute soft graviton amplitudes, we need the know the coupling of a soft graviton to the rest of the fields. For this we follow the strategy outlined in section 1:

1. First we write the metric as $\eta_{\mu \nu}+2 h_{\mu \nu}$ and expand the action in a power series in $h_{\mu \nu}$ and $\phi$. We shall use the Feynman rules derived from this action to compute amplitudes involving finite energy external particles but not external soft particles. For the analysis of this section we shall take the external states to be $\phi$ particles. 
2. In order to compute the amplitude involving a soft graviton of momentum $k$ and polarization $\varepsilon_{\mu \nu}$ satisfying ${ }^{3}$

$$
\eta_{\mu \nu} \varepsilon^{\mu \nu}=0, \quad \varepsilon^{\mu \nu}=\varepsilon^{\nu \mu}, \quad k_{\mu} \varepsilon^{\mu \nu}=0, \quad k_{\nu} \varepsilon^{\mu \nu}=0,
$$

we replace, in the action computed in step $1, \eta_{\mu \nu}$ by $\eta_{\mu \nu}+2 \varepsilon_{\mu \nu} e^{i k . x}$ and all ordinary derivatives by covariant derivatives computed with this metric and expand the action to first order in $\varepsilon_{\mu \nu}$. Since we have taken the polarization tensor to be traceless, we do not need to worry about the $\sqrt{\operatorname{det} g}$ term in the action. This determines the coupling of the soft graviton to the rest of the fields (scalars and finite energy gravitons) correctly to linear order in momentum $k^{\mu}$. There may be additional coupling at quadratic and higher order in $k^{\mu}$ through couplings involving Riemann tensor that are missed by this expansion.

We shall now proceed to evaluate the contribution from figure 1. Using the prescription given above, the coupling of the soft graviton to the scalar field is given by replacing $\eta^{\mu \nu}$ by $\eta^{\mu \nu}-2 \varepsilon^{\mu \nu} e^{i k . x}$ in the quadratic term in the action involving the scalar field:

$$
-\frac{1}{2} \int d^{D} x\left\{\eta^{\mu \nu} \partial_{\mu} \phi \partial_{\nu} \phi+M^{2} \phi^{2}\right\}
$$

We have assumed that the quadratic term contains only two derivatives. If it contained higher powers of $\left(-\square+M^{2}\right)$, they could be removed by a redefinition of $\phi$ that is regular near on-shell field configuration, and therefore would not affect the S-matrix. This gives the following coupling of the scalar to the soft graviton:

$$
\int d^{D} x \varepsilon^{\mu \nu} e^{i k \cdot x} \partial_{\mu} \phi \partial_{\nu} \phi
$$

Therefore the contribution from the three point vertex in figure 1 involving the soft graviton and the external scalar is given by

$$
2 i \varepsilon^{\mu \nu} p_{i \mu}\left(p_{i}+k\right)_{\nu}=2 i \varepsilon^{\mu \nu} p_{i \mu} p_{i \nu}
$$

where in the last step we have used (2.2). The second piece in figure 1 is the contribution from the scalar propagator carrying momentum $\left(p_{i}+k\right)$. This is given by

$$
-i \frac{1}{\left(p_{i}+k\right)^{2}+M^{2}}=-i \frac{1}{2 p_{i} \cdot k}
$$

using the on-shell conditions $p_{i}^{2}+M^{2}=0, k^{2}=0$. The final piece in figure 1 is the contribution from the amputated Green's function with external scalar particles of momenta $p_{1}, \ldots, p_{i-1}, p_{i}+k, p_{i+1}, \ldots, p_{N}$. This is given by

$$
\Gamma\left(p_{1}, \ldots, p_{N}\right)+k_{\rho} \frac{\partial}{\partial p_{i \rho}} \Gamma\left(p_{1}, \ldots, p_{N}\right)+\mathcal{O}\left(k_{\mu} k_{\nu}\right) .
$$

\footnotetext{
${ }^{3}$ Throughout our discussion all indices will be raised and lowered by $\eta^{\mu \nu}$ and $\eta_{\mu \nu}$.
} 
Taking the product of (2.5), (2.6) and (2.7) we get the full contribution from figure 1 to order unity. We can now sum over all diagrams in which the soft graviton is attached to any of the external states $i$. The net contribution from all these diagrams is given by

$$
\sum_{i=1}^{N} \varepsilon^{\mu \nu} p_{i \mu} p_{i \nu} \frac{1}{p_{i} \cdot k} \Gamma\left(p_{1}, \ldots, p_{N}\right)+\sum_{i=1}^{N} \varepsilon^{\mu \nu} p_{i \mu} p_{i \nu} \frac{1}{p_{i} \cdot k} k_{\rho} \frac{\partial}{\partial p_{i \rho}} \Gamma\left(p_{1}, \ldots, p_{N}\right)+\mathcal{O}(k) .
$$

The first term gives the amplitude to order $1 / k$, while the second term is of order unity. However if we want to compute the total order unity contribution, we also need to compute the contribution from the diagrams shown in figure 2 . In this figure $\widetilde{\Gamma}$ denotes the sum of Feynman diagrams from which external propagators have been removed, and also diagrams like the ones shown in figure 1 , in which by cutting a single internal propagator we can remove the soft line and one more external line from the rest of the diagram, have been removed. Therefore in figure 2 the soft graviton is attached to a hard internal line, and the amplitude has a finite limit as $k^{\mu} \rightarrow 0$. This in turn means that to evaluate these diagrams to order unity, we can set the momentum of the external soft graviton to zero. Using the rules for determining the soft graviton interaction vertex described earlier, we see that such an amplitude has the interpretation of a deformation of the amputated Green's function without the soft graviton under a constant change in the background metric $\eta^{\mu \nu} \rightarrow \eta^{\mu \nu}-2 \varepsilon^{\mu \nu}$ to first order in $\varepsilon_{\mu \nu}$. Since $\widetilde{\Gamma}\left(p_{1}, \ldots p_{N}\right)$ depends on the metric only via the combinations $g^{\mu \nu} p_{i \mu} p_{j \nu}=\left(\eta^{\mu \nu}-2 \varepsilon^{\mu \nu}\right) p_{i \mu} p_{j \nu}$, the effect of deforming the background metric by $2 \varepsilon_{\mu \nu}$ can also be equivalently represented by deforming $p_{i \mu}$ to $p_{i \mu}-\varepsilon_{\mu}^{\sigma} p_{i \sigma}$. Therefore the contribution from figure 2 can be expressed as

$$
-\sum_{i=1}^{N} \varepsilon_{\mu}^{\nu} p_{i \nu} \frac{\partial}{\partial p_{i \mu}} \Gamma\left(p_{1}, \ldots p_{N}\right) .
$$

Adding (2.8) and (2.9) we get the full amplitude to order unity:

$$
\begin{aligned}
\sum_{i=1}^{N} \varepsilon^{\mu \nu} p_{i \mu} p_{i \nu} & \frac{1}{p_{i} \cdot k} \Gamma\left(p_{1}, \ldots, p_{N}\right) \\
& +\sum_{i=1}^{N}\left[\varepsilon^{\mu \nu} p_{i \mu} p_{i \nu} \frac{1}{p_{i} \cdot k} k^{\rho}-\varepsilon_{\rho}^{\nu} p_{i \nu}\right] \frac{\partial}{\partial p_{i \rho}} \Gamma\left(p_{1}, \ldots, p_{N}\right)+\mathcal{O}(k) .
\end{aligned}
$$

This is the subleading soft graviton theorem for one external soft graviton [6, 45, 46].

Note that even if we set the external states on-shell by setting $p_{i}^{2}+M^{2}=0$, computation of $\partial \Gamma / \partial p_{i \rho}$ requires off-shell information. For example if we add to $\Gamma$ a contribution proportional to $\left(p_{i}^{2}+M^{2}\right)$ that vanishes on-shell, $\partial \Gamma / \partial p_{i \rho}$ gets a contribution proportional to $p_{i}^{\rho}$ that does not vanish on-shell. However when substituted into (2.10) it does vanish, showing that (2.10) depends only on on-shell data.

\section{One soft graviton theorem in tree level superstring field theory}

We now turn to superstring field theory [54]. It can be regarded as a regular field theory of infinite number of fields of arbitrarily high spin, with interaction vertices that are exponentially suppressed at large euclidean momenta. This makes the contribution from each 
Feynman graph manifestly ultraviolet finite, but otherwise the amplitudes are expressed as sum over Feynman diagrams just as an ordinary quantum field theory. Therefore as in section 2, the leading contribution to the soft graviton amplitude will come from figure 1 , and the subleading contribution will come from figure 1 and figure 2, although the Feynman rules for evaluating these diagrams will be different. We shall now evaluate these contributions by restricting the external states to be from the NS sector in the heterotic string theory and NSNS sector in type II string theory. During this analysis we shall allow for the possibility that some of the spatial directions have been compactified, and denote by $D$ the number of non-compact space-time dimensions.

Note that even though we make use of superstring field theory, at tree level amplitudes computed from this theory are identical to the standard amplitudes of superstring theory computed using world-sheet methods. Therefore our proof of subleading soft graviton theorem holds for the standard amplitudes computed using the world-sheet methods. At loop level the world-sheet approach gives divergent results when the masses of the external states are renormalized, but superstring field theory continues to give sensible S-matrix elements via the standard LSZ framework.

Before proceeding to the details of the analysis, let us comment on one underlying assumption that will be made in our analysis. In superstring field theory the graviton is a specific component of the string field. Therefore coupling of a soft graviton to the rest of the fields will be determined by the change in the interaction vertices / propagators due to the effect of switching on a low momentum plane wave solution of this field to first order in the field. We shall be using the fact that to linear order, this deformation is equivalent to a deformation of the world-sheet superconformal field theory underlying the construction of the superstring field theory due to a change in the target space metric $\eta_{\mu \nu}$ to $\eta_{\mu \nu}+2 \varepsilon_{\mu \nu} e^{i k . x}$. In the string field theory literature this property is known as background independence. Background independence of closed bosonic string field theory was established in [56, 57]. This has not yet been proved for superstring field theory, but there does not seem to be any specific difficulty in doing so [58]. Our analysis will assume background independence of superstring field theory, since we shall be computing the coupling of soft graviton by studying the effect of deforming the target space metric entering the construction of the world-sheet superconformal field theory.

Let us suppose that the $i$-th external particle is associated with some rank $n_{i}$ tensor field $\phi_{\mu_{1} \ldots \mu_{n_{i}}}$. In the Siegel gauge the kinetic operator acting on the NS sector states is proportional to $\left(L_{0}+\bar{L}_{0}\right)$ where $L_{n}, \bar{L}_{n}$ are the world-sheet Virasoro generators. Acting on a state of momentum $p$ and mass $M$ this is proportional to $p^{2}+M^{2}$. Therefore we can choose a basis for the NS sector string fields in which the kinetic term of $\phi_{\mu_{1} \ldots \mu_{n_{i}}}$ takes a particularly simple form ${ }^{4}$

$$
-\frac{1}{2} \int d^{D} x \prod_{j=1}^{n_{i}} \eta^{\mu_{j} \nu_{j}}\left[\eta^{\mu \nu} \partial_{\mu} \phi_{\mu_{1} \ldots \mu_{n_{i}}} \partial_{\nu} \phi_{\nu_{1} \ldots \nu_{n_{i}}}+M_{i}^{2} \phi_{\mu_{1} \ldots \mu_{n_{i}}} \phi_{\nu_{1} \ldots \nu_{n_{i}}}\right]
$$

\footnotetext{
${ }^{4}$ Note that we have taken the field $\phi$ to be a covariant tensor. While coupling it to a background soft graviton we shall take these to be the independent fields. This is of course related by field redefinition to the case where the fields with contravariant indices are regarded as independent fields. The S-matrix is independent of which prescription we choose.
} 
where $M_{i}$ denotes the tree level mass of the field $\phi_{\mu_{1} \ldots \mu_{n_{i}}}$. Typically the tensor has specific symmetry properties, but we can ignore this for now and restore it at the end by choosing the polarizations of the external states to have the required symmetry. Eq. (3.1) is the only specific property of the superstring field theory action that we shall use - we shall not need to use any detailed property of the interaction terms except general coordinate invariance of the action. From (3.1) we see that the coupling of the soft graviton to the tensor field $\phi$ has three types of terms:

1. The first type of term is given by the replacement of $\eta^{\mu \nu}$ by $\eta^{\mu \nu}-2 \varepsilon^{\mu \nu} e^{i k . x}$ in (3.1). The effect of this is identical to that given in (2.5), and generates a contribution to the vertex:

$$
2 i \varepsilon^{\mu \nu} p_{i \mu} p_{i \nu} \prod_{j=1}^{n_{i}} \eta^{\mu_{j} \nu_{j}}
$$

where $\mu_{1}, \ldots, \mu_{n_{i}}$ are the Lorentz indices carried by the external state carrying momentum $p_{i}$ and $\nu_{1}, \ldots, \nu_{n_{i}}$ are the Lorentz indices carried by the internal state carrying momentum $p_{i}+k$.

2. The second kind of contribution comes from replacing in (3.1) the $\eta^{\mu_{\ell} \nu_{\ell}}$ factor by $\eta^{\mu_{\ell} \nu_{\ell}}-2 \varepsilon^{\mu_{\ell} \nu_{\ell}} e^{i k . x}$ for $1 \leq \ell \leq n_{i}$. The effect of this is to generate an interaction vertex

$$
2 i\left\{\eta^{\mu \nu} p_{i \mu}\left(p_{i}+k\right)_{\nu}+M_{i}^{2}\right\} \sum_{\ell=1}^{n_{i}} \varepsilon^{\mu_{\ell} \nu_{\ell}} \prod_{\substack{j=1 \\ j \neq \ell}}^{n_{i}} \eta^{\mu_{j} \nu_{j}}=2 i p_{i} \cdot k \sum_{\ell=1}^{n_{i}} \varepsilon^{\mu_{\ell} \nu_{\ell}} \prod_{\substack{j=1 \\ j \neq \ell}}^{n_{i}} \eta^{\mu_{j} \nu_{j}},
$$

where in the second step we have used the on-shell condition $p_{i}^{2}+M_{i}^{2}=0$.

3. The third type of contribution comes from replacing the $\partial_{\mu} \phi_{\mu_{1} \ldots \mu_{n_{i}}}\left(\right.$ or $\left.\partial_{\nu} \phi_{\nu_{1} \ldots \nu_{n_{i}}}\right)$ factor by

$$
\begin{aligned}
D_{\mu} \phi_{\mu_{1} \ldots \mu_{n_{i}}}= & \partial_{\mu} \phi_{\mu_{1} \ldots \mu_{n_{i}}}-\sum_{\ell=1}^{n_{i}} \Gamma_{\mu \mu_{\ell}}^{\rho_{\ell}} \phi_{\mu_{1} \ldots \mu_{\ell-1} \rho_{\ell} \mu_{\ell+1} \ldots \mu_{n_{i}}} \\
= & \partial_{\mu} \phi_{\mu_{1} \ldots \mu_{n_{i}}}-i e^{i k . x} \sum_{\ell=1}^{n_{i}}\left(k_{\mu} \varepsilon_{\mu_{\ell}}^{\rho_{\ell}}+k_{\mu_{\ell}} \varepsilon_{\mu}^{\rho_{\ell}}-k^{\rho_{\ell}} \varepsilon_{\mu \mu_{\ell}}\right) \phi_{\mu_{1} \ldots \mu_{\ell-1} \rho_{\ell} \mu_{\ell+1} \ldots \mu_{n_{i}}} \\
& +\mathcal{O}\left(k_{\rho} k_{\sigma}\right)
\end{aligned}
$$

This, together with similar expression for $D_{\nu} \phi_{\nu_{1} \ldots \nu_{n}}$, generates the following net contribution to the vertex to first order in the soft momenta:

$$
\begin{aligned}
i \eta^{\mu \nu}\left(p_{i}+k\right)_{\nu} & \sum_{\ell=1}^{n_{i}}\left(k_{\mu} \varepsilon^{\mu_{\ell} \nu_{\ell}}+k^{\nu_{\ell}} \varepsilon_{\mu}^{\mu_{\ell}}-k^{\mu_{\ell}} \varepsilon_{\mu}^{\nu_{\ell}}\right) \prod_{\substack{j=1 \\
j \neq \ell}}^{n_{i}} \eta^{\mu_{j} \nu_{j}} \\
& -i \eta^{\mu \nu} p_{i \mu} \sum_{\ell=1}^{n_{i}}\left(k_{\nu} \varepsilon^{\nu_{\ell} \mu_{\ell}}+k^{\mu_{\ell}} \varepsilon_{\nu}^{\nu_{\ell}}-k^{\nu_{\ell}} \varepsilon_{\nu}^{\mu_{\ell}}\right) \prod_{\substack{j=1 \\
j \neq \ell}}^{n_{i}} \eta^{\mu_{j} \nu_{j}}
\end{aligned}
$$

Here $\mu_{1}, \ldots \mu_{n_{i}}$ are the Lorentz indices carried by the external line of momentum $p_{i}$ and $\nu_{1}, \ldots, \nu_{i}$ are the Lorentz indices carried by the internal line of momentum $p_{i}+k$. 
Adding (3.2), (3.3) and (3.5) and keeping terms up to order $k$ we get the following net contribution to the three point vertex of figure 1 with one soft graviton carrying momentum $k$ and a pair of $\phi$ particles carrying momenta $p_{i}$ and $-p_{i}-k$ :

$$
2 i \varepsilon^{\mu \nu} p_{i \mu} p_{i \nu} \prod_{j=1}^{n_{i}} \eta^{\mu_{j} \nu_{j}}+2 i \sum_{\ell=1}^{n_{i}}\left[p_{i} \cdot k \varepsilon^{\mu_{\ell} \nu_{\ell}}-p_{i \mu} \varepsilon^{\mu \nu_{\ell}} k^{\mu_{\ell}}+p_{i \mu} \varepsilon^{\mu \mu_{\ell}} k^{\nu_{\ell}}\right] \prod_{\substack{j=1 \\ j \neq \ell}}^{n_{i}} \eta^{\mu_{j} \nu_{j}}+\mathcal{O}\left(k_{\rho} k_{\sigma}\right) .
$$

The next task is to compute the $\phi$ propagator carrying momentum $p_{i}+k$. This is easily read from (3.1) to be

$$
-i \prod_{j=1}^{n_{i}} \eta_{\nu_{j} \rho_{j}} \frac{1}{\left(p_{i}+k\right)^{2}+M^{2}}=-i \prod_{j=1}^{n_{i}} \eta_{\nu_{j} \rho_{j}} \frac{1}{2 p_{i} \cdot k}
$$

where $\rho_{1}, \ldots, \rho_{n_{i}}$ are the tensor indices carried by the right end of the internal propagator carrying momentum $p_{i}+k$.

Finally we turn to the part of the amplitude $\Gamma$ with external states carrying momenta $p_{1}, \ldots, p_{i-1}, p_{i}+k, p_{i+1}, \ldots, p_{N}$. If $\rho_{1} \ldots, \rho_{n_{i}}$ are the tensor indices carried by the leg of momentum $p_{i}+k$ entering this amplitude, then we shall denote the amplitude by $\Gamma^{\rho_{1} \ldots \rho_{n_{i}}}$, suppressing the tensor indices associated with the other external states of $\Gamma . \Gamma^{\rho_{1} \ldots \rho_{n_{i}}}$ may be expressed as

$$
\begin{aligned}
\Gamma^{\rho_{1} \ldots \rho_{n_{i}}} & \left(p_{1}, \ldots, p_{i-1}, p_{i}+k, p_{i+1}, \ldots, p_{N}\right) \\
& =\Gamma^{\rho_{1} \ldots \rho_{n_{i}}}\left(p_{1}, \ldots, p_{N}\right)+k_{\sigma} \frac{\partial}{\partial p_{i \sigma}} \Gamma^{\rho_{1} \ldots \rho_{n_{i}}}\left(p_{1}, \ldots, p_{N}\right)+\mathcal{O}\left(k_{\mu} k_{\nu}\right) .
\end{aligned}
$$

The net contribution to figure 1 to order unity is now obtained by taking the product of (3.6), (3.7), (3.8) and the polarization tensor $\epsilon_{\mu_{1} \ldots \mu_{n_{i}}}$ of the external $\phi$ field, expanding the result to order unity and finally summing over $i$. This gives

$$
\begin{aligned}
& \sum_{i=1}^{N} \varepsilon^{\mu \nu} p_{i \mu} p_{i \nu} \frac{1}{p_{i} \cdot k} \epsilon_{\mu_{1} \ldots \mu_{n_{i}}} \Gamma^{\mu_{1} \ldots \mu_{n_{i}}}+\sum_{i=1}^{N} \varepsilon^{\mu \nu} p_{i \mu} p_{i \nu} \frac{1}{p_{i} \cdot k} \epsilon_{\mu_{1} \ldots \mu_{n_{i}}} k_{\sigma} \frac{\partial}{\partial p_{i \sigma}} \Gamma^{\mu_{1} \ldots \mu_{n_{i}}} \\
& +\sum_{i=1}^{N} \epsilon_{\mu_{1} \ldots \mu_{n_{i}}} \sum_{\ell=1}^{n_{i}} \frac{1}{p_{i} \cdot k}\left[p_{i} \cdot k \varepsilon_{\nu_{\ell}}^{\mu_{\ell}}-p_{i \mu} \varepsilon^{\mu}{ }_{\nu \ell} k^{\mu_{\ell}}+p_{i \mu} \varepsilon^{\mu \mu_{\ell}} k_{\nu_{\ell}}\right] \Gamma^{\mu_{1} \ldots \mu_{\ell-1} \nu_{\ell} \mu_{\ell+1} \ldots \mu_{n_{i}}}
\end{aligned}
$$

To this we have to add the leading contribution from figure 2. This is given by the change in the amplitude $\epsilon_{\mu_{1} \ldots \mu_{n_{i}}} \Gamma^{\mu_{1} \ldots \mu_{n_{i}}}$ under the variation $\eta^{\rho \sigma} \rightarrow \eta^{\rho \sigma}-2 \varepsilon^{\rho \sigma}$. Since the final expression for the amplitude is given by products of $\epsilon_{\mu_{1} \ldots \mu_{n_{i}}}$ 's and $p_{i \mu}$ 's contracted with various factors of $\eta^{\rho \sigma}$, changing $\eta^{\rho \sigma}$ to $\eta^{\rho \sigma}-2 \varepsilon^{\rho \sigma}$ can also be implemented by the change

$$
p_{\mu} \rightarrow p_{\mu}-\varepsilon_{\mu}^{\nu} p_{\nu}, \quad \epsilon_{\mu_{1} \ldots \mu_{n_{i}}} \rightarrow \epsilon_{\mu_{1} \ldots \mu_{n_{i}}}-\sum_{\ell=1}^{n_{i}} \varepsilon_{\mu_{\ell}}^{\nu_{\ell}} \epsilon_{\mu_{1} \ldots \mu_{\ell-1} \nu_{\ell} \mu_{\ell+1} \ldots \mu_{n_{i}}}
$$




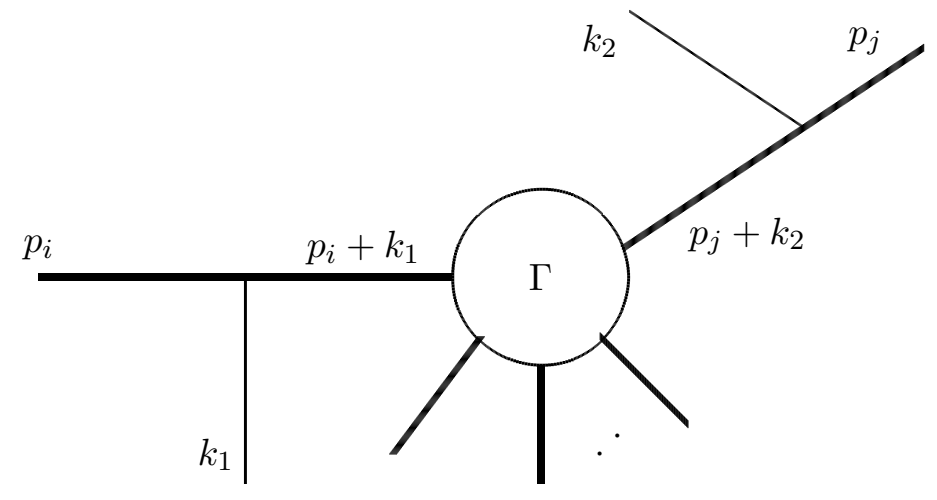

Figure 3. Two external soft gravitons attached to different external lines carrying finite momenta.

This determines the amplitude given in figure 2 to be

$$
-\sum_{i=1}^{N} \varepsilon_{\mu}^{\nu} \epsilon_{\mu_{1} \ldots \mu_{n_{i}}} p_{i \nu} \frac{\partial}{\partial p_{i \mu}} \Gamma^{\mu_{1} \ldots \mu_{n_{i}}}\left(p_{1}, \ldots, p_{N}\right)-\sum_{i=1}^{N} \sum_{\ell=1}^{n_{i}} \varepsilon_{\mu_{\ell}}^{\nu_{\ell}} \epsilon_{\mu_{1} \ldots \mu_{\ell-1} \nu_{\ell} \mu_{\ell+1} \ldots \mu_{n_{i}}} \Gamma^{\mu_{1} \ldots \mu_{n_{i}}} .
$$

Adding (3.9) to (3.11) we now get the full contribution to the amplitude of one soft graviton and $N$ finite energy particles to the first subleading order in the soft momentum:

$$
\begin{aligned}
{\left[\sum_{i=1}^{N} \varepsilon^{\mu \nu} p_{i \mu} p_{i \nu} \frac{1}{p_{i} \cdot k}\right] \Gamma\left(p_{1}, \ldots, p_{N}\right) } \\
\quad+\sum_{i=1}^{N} \epsilon_{\mu_{1} \ldots \mu_{n_{i}}}\left(\varepsilon^{\mu \nu} p_{i \mu} p_{i \nu} k_{\sigma} \frac{1}{p_{i} \cdot k}-\varepsilon_{\sigma}^{\nu} p_{i \nu}\right) \frac{\partial}{\partial p_{i \sigma}} \Gamma^{\mu_{1} \ldots \mu_{n_{i}}} \\
\quad-\sum_{i=1}^{N} \frac{1}{p_{i} \cdot k} \epsilon_{\mu_{1} \ldots \mu_{n_{i}}} \sum_{\ell=1}^{n_{i}}\left[p_{i \mu} \varepsilon^{\mu}{ }_{\nu_{\ell}} k^{\mu_{\ell}}-p_{i \mu} \varepsilon^{\mu \mu_{\ell}} k_{\nu_{\ell}}\right] \Gamma^{\mu_{1} \ldots \mu_{\ell-1} \nu_{\ell} \mu_{\ell+1} \ldots \mu_{n_{i}}}
\end{aligned}
$$

In the first term $\Gamma$ denotes the amplitude with the same external finite energy states but without soft graviton insertion - being equal to $\epsilon_{\mu_{1} \ldots \mu_{n_{i}}} \Gamma^{\mu_{1} \ldots \mu_{n_{i}}}$ in the notation of (3.11). In the second and the third line it is understood that in the $i$-th term inside the sum, we have suppressed the polarization tensor of all states other than the $i$-th state and the corresponding indices of $\Gamma$.

Eq. (3.12) is the soft graviton theorem to first subleading order [6, 45, 46].

\section{Multiple soft gravitons}

We shall now consider amplitudes with multiple soft gravitons but restrict our analysis to the leading order in the soft momenta. This analysis will be identical to that in [2]. Consider first the case where we have two soft external gravitons carrying momenta $k_{1}$ and $k_{2}$. The maximum power of soft momenta in the denominator of such an amplitude is two. This can arise from the two soft gravitons attaching on different external legs as 


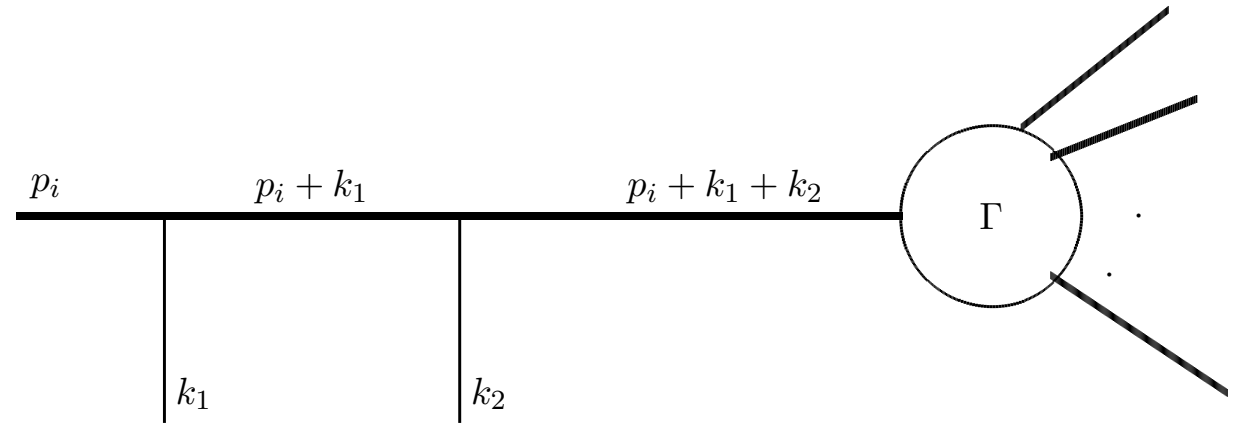

Figure 4. Two external soft gravitons attached to the same external line carrying finite momenta.

in figure 3 or both soft gravitons attaching on the same external leg as in figure 4 . In both diagrams, we can compute the product of the leading contributions from the three point vertex from (3.2) and the propagator that follows it from (3.7). When the two soft gravitons attach to different external lines as in figure 3 , the amplitude takes the form

$$
\frac{1}{p_{i} \cdot k_{1}} \varepsilon_{\mu \nu}^{(1)} p_{i}^{\mu} p_{i}^{\nu} \times \frac{1}{p_{j} \cdot k_{2}} \varepsilon_{\rho \sigma}^{(2)} p_{j}^{\rho} p_{j}^{\sigma} \times \Gamma^{(N)}\left(p_{1}, \ldots, p_{N}\right)+\text { less singular terms }
$$

where $\Gamma^{(N)}\left(p_{1}, \ldots, p_{N}\right)$ denotes the amplitude without soft gravitons with all indices contracted with the external polarization tensors. On the other hand when both soft lines attach to the same external line as in figure 4 the amplitude takes the form

$$
\frac{1}{p_{i} \cdot k_{1}} \varepsilon_{\mu \nu}^{(1)} p_{i}^{\mu} p_{i}^{\nu} \times \frac{1}{p_{i} \cdot\left(k_{1}+k_{2}\right)} \varepsilon_{\rho \sigma}^{(2)} p_{j}^{\rho} p_{j}^{\sigma} \times \Gamma^{(N)}\left(p_{1}, \ldots, p_{N}\right)+\text { less singular terms } .
$$

Adding to this another contribution where the external soft lines carrying momenta $k_{1}$ and $k_{2}$ are exchanged we get

$$
\frac{1}{p_{i} \cdot k_{1}} \varepsilon_{\mu \nu}^{(1)} p_{i}^{\mu} p_{i}^{\nu} \times \frac{1}{p_{i} \cdot k_{2}} \varepsilon_{\rho \sigma}^{(2)} p_{i}^{\rho} p_{i}^{\sigma} \times \Gamma^{(N)}\left(p_{1}, \ldots, p_{N}\right)+\text { less singular terms } .
$$

Summing over all possible insertions of the two soft lines on $N$ external lines carrying finite momentum, we now get [2]

$$
\sum_{i=1}^{N} \frac{1}{p_{i} \cdot k_{1}} \varepsilon_{\mu \nu}^{(1)} p_{i}^{\mu} p_{i}^{\nu} \times \sum_{j=1}^{N} \frac{1}{p_{j} \cdot k_{2}} \varepsilon_{\rho \sigma}^{(2)} p_{j}^{\rho} p_{j}^{\sigma} \times \Gamma^{(N)}\left(p_{1}, \ldots, p_{N}\right)+\text { less singular terms } .
$$

If we have $m$ external soft gravitons then the leading soft term will have $m$ powers of soft momentum in the denominator. For this each external soft particle must attach to a nearly on-shell line. We again sum over all possible insertions, including multiple insertions on a single external line in all possible order. This leads to a generalization of (4.4) of the form:

$$
\begin{aligned}
& \Gamma^{(N+m)}\left(\varepsilon^{(1)}, k_{1}, \ldots, \varepsilon^{(m)}, k_{m} ; p_{1}, \ldots, p_{N}\right)= \prod_{\substack{\alpha=1 \\
\\
\\
+\text { less singular terms }}}^{m}\left[\sum_{i=1}^{N} \frac{1}{p_{i} \cdot k_{\alpha}} \varepsilon_{\mu \nu}^{(\alpha)} p_{i}^{\mu} p_{i}^{\nu}\right] \Gamma^{(N)}\left(p_{1}, \ldots, p_{N}\right) \\
&
\end{aligned}
$$




\section{Generalizations}

In this section we shall discuss possible generalizations of our result. One immediate generalization is the derivation of the leading soft photon theorem for superstring tree amplitudes for arbitrary number of external states [55]. The analysis is very similar to that in section 4 . We determine the leading order coupling of a soft photon of polarization $\varepsilon_{\mu}$ by replacing the momentum $q_{\mu}$ by $q_{\mu}-Q \varepsilon_{\mu}$ in the expression for the kinetic term. Here $Q$ denotes the charge carried by the particle. This gives the analog of (3.2):

$$
2 i Q \varepsilon^{\mu} p_{i \mu} \prod_{j=1}^{n_{i}} \eta^{\mu_{j} \nu_{j}}
$$

Using this we can follow the same procedure as given in section 3, section 4 to derive the multiple leading soft photon theorem for $m$ soft photons of polarizations $\varepsilon^{(1)}, \ldots, \varepsilon^{(m)}$ and momenta $k_{1}, \ldots, k_{m}$ attached to an amplitude with $N$ finite energy particles:

$$
\begin{aligned}
& \Gamma^{(N+m)}\left(\varepsilon^{(1)}, k_{1}, \ldots, \varepsilon^{(m)}, k_{m} ; p_{1}, \ldots, p_{N}\right)=\prod_{\alpha=1}^{m}\left[\sum_{i=1}^{N} \frac{1}{p_{i} \cdot k_{\alpha}} Q_{i} \varepsilon_{\mu}^{(\alpha)} p_{i}^{\mu}\right] \Gamma^{(N)}\left(p_{1}, \ldots, p_{N}\right) \\
& \text { +less singular terms. }
\end{aligned}
$$

For soft photons, we do not expect any universal result beyond the leading term since soft photons can couple to the rest of the fields via non-minimal coupling involving the field strength, and these interactions cost only one power of soft momentum. Therefore (5.2) gives the most general universal soft photon theorem.

A more interesting generalization would be to extend the analysis of section 3 to derive the subleading soft graviton theorem to all orders in string perturbation theory for all finite energy external states. For this let us restrict the discussion to the cases where the number of non-compact space-time dimensions is five or more so that the amplitudes are free from infrared divergences. ${ }^{5}$ In this case the contribution to the amplitude with $N$ finite energy external particles and one soft graviton will still be given by the sum of the contributions shown in figure 1 and figure 2, but in figure 1 the three point vertex describing the coupling of the soft graviton to the finite energy particle is now the full 1PI vertex and the internal propagator carrying momentum $p_{i}+k$ is now the full (finitely) renormalized propagator, and in figure 2 the blob labelled $\widetilde{\Gamma}$ now denotes the full Green's function with external propagators removed and the contributions of the form given in figure 1 subtracted. The subleading contribution coming from figure 2 will continue to have the same form as given in (3.11). ${ }^{6}$ The main difficulty is in the determination of the contribution to figure 1 to the first subleading order, since the renormalized two point function does not have the simple

\footnotetext{
${ }^{5}$ In four space-time dimensions the subleading soft graviton theorem is known to be corrected due to infrared divergences [8].

${ }^{6}$ In this context note that even though we have used a notation in which the tensor fields carry space-time indices, we could have also used a notation in which they carry flat tangent space indices by multiplying the tensors by the symmetric square root of the inverse metric for each index and treating these as independent field variables. This would make some of the intermediate steps in the analysis different, e.g. in (3.11) the second term will be absent and in (3.4) we would have to use the spin connection instead of Christoffel symbol
} 
form given in (3.1) and consequently the coupling of the soft graviton to the finite energy particles also have a more complicated form. A promising avenue will be to try to show that the quadratic term in the fields in the 1PI effective action [54] of superstring field theory can be brought to the diagonal form given in (3.1) after a field redefinition. In that case we can use this 1PI effective action to derive the subleading soft graviton theorem following the analysis of section 3 . This should certainly be possible for scalar fields, but it is not clear if this can also be achieved for general tensor fields. Another possibility - which should also apply to Ramond sector external states - will be to work with the renormalized kinetic term as given in [54], but use the gauge invariance of the 1PI effective action to show that the general results of section 3 still hold.

In this context it is useful to note that the contribution to figure 2 given in (3.11) takes the form of a differential operator acting on the amplitude without the soft particle, and that the differential operator is 'local' in the sense that it contains a sum of terms each of which involves the soft momentum and the momentum of one finite energy external state. The contributions from figure 1 also will have this form. These were the key ingredients based on which [13] determined the form of the subleading soft graviton theorem up to some overall normalization constants multiplying the terms that act non-trivially on the polarization tensors of the external particles. The new feature here is the possibility of mixing between states carrying different tensor indices at the same mass level, including mixing between physical and unphysical states. Therefore it is conceivable that by gaining some basic knowledge of the contribution to figure 1 involving the soft graviton coupling to the external state and the renormalized propagator one will be able to prove the subleading soft graviton theorem for these amplitudes. Such arguments should be applicable to all external finite energy states, both in the NS and R-sector.

\section{Acknowledgments}

This work was supported in part by the DAE project 12-R\&D-HRI-5.02-0303 and J. C. Bose fellowship of the Department of Science and Technology, India.

Open Access. This article is distributed under the terms of the Creative Commons Attribution License (CC-BY 4.0), which permits any use, distribution and reproduction in any medium, provided the original author(s) and source are credited.

\section{References}

[1] S. Weinberg, Photons and gravitons in $S$ matrix theory: derivation of charge conservation and equality of gravitational and inertial mass, Phys. Rev. 135 (1964) B1049 [InSPIRE].

[2] S. Weinberg, Infrared photons and gravitons, Phys. Rev. 140 (1965) B516 [InSPIRE].

[3] D.J. Gross and R. Jackiw, Low-energy theorem for graviton scattering, Phys. Rev. 166 (1968) 1287 [INSPIRE].

for defining covariant derivatives. However the final result would remain unchanged and this formalism would also be suitable for application to states carrying spinor indices, at least for the evaluation of the contribution from figure 2 . 
[4] R. Jackiw, Low-energy theorems for massless bosons: photons and gravitons, Phys. Rev. 168 (1968) 1623 [INSPIRE].

[5] C.D. White, Factorization properties of soft graviton amplitudes, JHEP 05 (2011) 060 [arXiv:1103.2981] [INSPIRE].

[6] F. Cachazo and A. Strominger, Evidence for a new soft graviton theorem, arXiv:1404.4091 [INSPIRE].

[7] B.U.W. Schwab and A. Volovich, Subleading soft theorem in arbitrary dimensions from scattering equations, Phys. Rev. Lett. 113 (2014) 101601 [arXiv:1404.7749] [INSPIRE].

[8] Z. Bern, S. Davies and J. Nohle, On loop corrections to subleading soft behavior of gluons and gravitons, Phys. Rev. D 90 (2014) 085015 [arXiv: 1405.1015] [INSPIRE].

[9] S. He, Y.-t. Huang and C. Wen, Loop corrections to soft theorems in gauge theories and gravity, JHEP 12 (2014) 115 [arXiv: 1405.1410] [INSPIRE].

[10] A.J. Larkoski, Conformal invariance of the subleading soft theorem in gauge theory, Phys. Rev. D 90 (2014) 087701 [arXiv:1405.2346] [INSPIRE].

[11] F. Cachazo and E.Y. Yuan, Are soft theorems renormalized?, arXiv:1405.3413 [INSPIRE].

[12] N. Afkhami-Jeddi, Soft graviton theorem in arbitrary dimensions, arXiv:1405.3533 [INSPIRE].

[13] J. Broedel, M. de Leeuw, J. Plefka and M. Rosso, Constraining subleading soft gluon and graviton theorems, Phys. Rev. D 90 (2014) 065024 [arXiv:1406.6574] [INSPIRE].

[14] Z. Bern, S. Davies, P. Di Vecchia and J. Nohle, Low-energy behavior of gluons and gravitons from gauge invariance, Phys. Rev. D 90 (2014) 084035 [arXiv: 1406.6987] [InSPIRE].

[15] C.D. White, Diagrammatic insights into next-to-soft corrections, Phys. Lett. B 737 (2014) 216 [arXiv: 1406.7184] [INSPIRE].

[16] M. Zlotnikov, Sub-sub-leading soft-graviton theorem in arbitrary dimension, JHEP 10 (2014) 148 [arXiv: 1407.5936] [INSPIRE].

[17] C. Kalousios and F. Rojas, Next to subleading soft-graviton theorem in arbitrary dimensions, JHEP 01 (2015) 107 [arXiv: 1407.5982] [INSPIRE].

[18] Y.-J. Du, B. Feng, C.-H. Fu and Y. Wang, Note on soft graviton theorem by KLT relation, JHEP 11 (2014) 090 [arXiv: 1408.4179] [INSPIRE].

[19] D. Bonocore, E. Laenen, L. Magnea, L. Vernazza and C.D. White, The method of regions and next-to-soft corrections in Drell-Yan production, Phys. Lett. B 742 (2015) 375 [arXiv: 1410.6406] [INSPIRE].

[20] A. Sabio Vera and M.A. Vazquez-Mozo, The double copy structure of soft gravitons, JHEP 03 (2015) 070 [arXiv: 1412.3699] [INSPIRE].

[21] F. Cachazo, S. He and E.Y. Yuan, New double soft emission theorems, Phys. Rev. D 92 (2015) 065030 [arXiv:1503.04816] [INSPIRE].

[22] A.E. Lipstein, Soft theorems from conformal field theory, JHEP 06 (2015) 166 [arXiv: 1504.01364] [INSPIRE].

[23] S.D. Alston, D.C. Dunbar and W.B. Perkins, n-point amplitudes with a single negative-helicity graviton, Phys. Rev. D 92 (2015) 065024 [arXiv:1507.08882] [INSPIRE]. 
[24] Y.-t. Huang and C. Wen, Soft theorems from anomalous symmetries, JHEP 12 (2015) 143 [arXiv: 1509.07840] [INSPIRE].

[25] J. Rao and B. Feng, Note on identities inspired by new soft theorems, JHEP 04 (2016) 173 [arXiv: 1604.00650] [INSPIRE].

[26] F. Cachazo, P. Cha and S. Mizera, Extensions of theories from soft limits, JHEP 06 (2016) 170 [arXiv: 1604.03893] [INSPIRE].

[27] A.P. Saha, Double soft theorem for perturbative gravity, JHEP 09 (2016) 165 [arXiv: 1607.02700] [INSPIRE].

[28] A. Luna, S. Melville, S.G. Naculich and C.D. White, Next-to-soft corrections to high energy scattering in QCD and gravity, JHEP 01 (2017) 052 [arXiv:1611.02172] [INSPIRE].

[29] C. Cheung, K. Kampf, J. Novotny, C.-H. Shen and J. Trnka, A periodic table of effective field theories, JHEP 02 (2017) 020 [arXiv:1611.03137] [INSPIRE].

[30] H. Elvang, C.R.T. Jones and S.G. Naculich, Soft photon and graviton theorems in effective field theory, Phys. Rev. Lett. 118 (2017) 231601 [arXiv:1611.07534] [INSPIRE].

[31] A.P. Saha, Double soft theorem for perturbative gravity II: some details on CHY soft limits, arXiv: 1702.02350 [INSPIRE].

[32] M. Ademollo et al., Soft dilations and scale renormalization in dual theories, Nucl. Phys. B 94 (1975) 221 [INSPIRE].

[33] J.A. Shapiro, On the renormalization of dual models, Phys. Rev. D 11 (1975) 2937 [InSPIRE].

[34] B.U.W. Schwab, Subleading soft factor for string disk amplitudes, JHEP 08 (2014) 062 [arXiv: 1406.4172] [INSPIRE].

[35] M. Bianchi, S. He, Y.-t. Huang and C. Wen, More on soft theorems: trees, loops and strings, Phys. Rev. D 92 (2015) 065022 [arXiv:1406.5155] [INSPIRE].

[36] B.U.W. Schwab, A note on soft factors for closed string scattering, JHEP 03 (2015) 140 [arXiv: 1411.6661] [INSPIRE].

[37] P. Di Vecchia, R. Marotta and M. Mojaza, Soft theorem for the graviton, dilaton and the Kalb-Ramond field in the bosonic string, JHEP 05 (2015) 137 [arXiv:1502.05258] [INSPIRE].

[38] M. Bianchi and A.L. Guerrieri, On the soft limit of open string disk amplitudes with massive states, JHEP 09 (2015) 164 [arXiv: 1505.05854] [INSPIRE].

[39] S.G. Avery and B.U.W. Schwab, Burg-Metzner-Sachs symmetry, string theory and soft theorems, Phys. Rev. D 93 (2016) 026003 [arXiv:1506.05789] [INSPIRE].

[40] A.L. Guerrieri, Soft behavior of string amplitudes with external massive states, Nuovo Cim. C 39 (2016) 221 [arXiv:1507.08829] [INSPIRE].

[41] P. Di Vecchia, R. Marotta and M. Mojaza, Soft theorems from string theory, Fortsch. Phys. 64 (2016) 389 [arXiv:1511.04921] [INSPIRE].

[42] M. Bianchi and A.L. Guerrieri, On the soft limit of tree-level string amplitudes, arXiv: 1601.03457 [INSPIRE].

[43] P. Di Vecchia, R. Marotta and M. Mojaza, Subsubleading soft theorems of gravitons and dilatons in the bosonic string, JHEP 06 (2016) 054 [arXiv: 1604.03355] [INSPIRE]. 
[44] P. Di Vecchia, R. Marotta and M. Mojaza, Soft behavior of a closed massless state in superstring and universality in the soft behavior of the dilaton, JHEP 12 (2016) 020 [arXiv: 1610.03481] [INSPIRE].

[45] A. Strominger, On BMS invariance of gravitational scattering, JHEP 07 (2014) 152 [arXiv: 1312.2229] [INSPIRE].

[46] T. He, V. Lysov, P. Mitra and A. Strominger, BMS supertranslations and Weinberg's soft graviton theorem, JHEP 05 (2015) 151 [arXiv: 1401.7026] [INSPIRE].

[47] A. Strominger and A. Zhiboedov, Gravitational memory, BMS supertranslations and soft theorems, JHEP 01 (2016) 086 [arXiv:1411.5745] [INSPIRE].

[48] M. Campiglia and A. Laddha, Asymptotic symmetries of gravity and soft theorems for massive particles, JHEP 12 (2015) 094 [arXiv: 1509.01406] [INSPIRE].

[49] M. Campiglia and A. Laddha, Sub-subleading soft gravitons: new symmetries of quantum gravity?, Phys. Lett. B 764 (2017) 218 [arXiv:1605.09094] [INSPIRE].

[50] E. Conde and P. Mao, BMS supertranslations and not so soft gravitons, JHEP 05 (2017) 060 [arXiv: 1612.08294] [INSPIRE].

[51] T. He, D. Kapec, A.-M. Raclariu and A. Strominger, Loop-corrected virasoro symmetry of $4 D$ quantum gravity, arXiv: 1701.00496 [INSPIRE].

[52] M. Campiglia and A. Laddha, Sub-subleading soft gravitons and large diffeomorphisms, JHEP 01 (2017) 036 [arXiv: 1608.00685] [INSPIRE].

[53] M. Asorey, A.P. Balachandran, F. Lizzi and G. Marmo, Equations of motion as constraints: superselection rules, ward identities, JHEP 03 (2017) 136 [arXiv: 1612.05886] [INSPIRE].

[54] C. de Lacroix, H. Erbin, S.P. Kashyap, A. Sen and M. Verma, Closed superstring field theory and its applications, arXiv:1703.06410 [INSPIRE].

[55] F.E. Low, Bremsstrahlung of very low-energy quanta in elementary particle collisions, Phys. Rev. 110 (1958) 974 [INSPIRE].

[56] A. Sen and B. Zwiebach, A proof of local background independence of classical closed string field theory, Nucl. Phys. B 414 (1994) 649 [hep-th/9307088] [InSPIRE].

[57] A. Sen and B. Zwiebach, Quantum background independence of closed string field theory, Nucl. Phys. B 423 (1994) 580 [hep-th/9311009] [INSPIRE].

[58] A. Sen, work in progress. 